\title{
Structural Brain Changes in Patients With COPD
}

Roland W. Esser, Dipl-Psych; M. Cornelia Stoeckel, PhD; Anne Kirsten, MD; Henrik Watz, MD; Karin Taube, MD; Kirsten Lehmann; Sibylle Petersen, PhD; Helgo Magnussen, MD; and Andreas von Leupoldt, PhD

BACKGROUND: Patients with COPD suffer from chronic dyspnea, which is commonly perceived as highly aversive and threatening. Moreover, COPD is often accompanied by disease-specific fears and avoidance of physical activity. However, little is known about structural brain changes in patients with COPD and respective relations with disease duration and disease-specific fears.

METHODS: This study investigated structural brain changes in patients with COPD and their relation with disease duration, fear of dyspnea, and fear of physical activity. We used voxelbased morphometric analysis of MRI images to measure differences in generalized cortical degeneration and regional gray matter between 30 patients with moderate to severe COPD and 30 matched healthy control subjects. Disease-specific fears were assessed by the COPD anxiety questionnaire.

RESULTS: Patients with COPD showed no generalized cortical degeneration, but decreased gray matter in posterior cingulate cortex (whole-brain analysis) as well as in anterior and midcingulate cortex, hippocampus, and amygdala (regions-of-interest analyses). Patients' reductions in gray matter in anterior cingulate cortex were negatively correlated with disease duration, fear of dyspnea, and fear of physical activity. Mediation analysis revealed that the relation between disease duration and reduced gray matter of the anterior cingulate was mediated by fear of physical activity.

CONCLUSIONS: Patients with COPD demonstrated gray matter decreases in brain areas relevant for the processing of dyspnea, fear, and antinociception. These structural brain changes were partly related to longer disease duration and greater disease-specific fears, which might contribute to a less favorable course of the disease.

CHEST 2016; 149(2):426-434

KEY WORDS: COPD; dyspnea; MRI; psychology

ABBREVIATIONS: $\mathrm{ACC}=$ anterior cingulate cortex; $\mathrm{ACC}-\mathrm{GMV}=$ gray matter volume in the anterior cingulate cortex; AMYG = amygdala; CAF = COPD Anxiety Questionnaire; DARTEL = diffeomorphic anatomical registration using exponentiated lie algebra; FWE-svc $=$ family-wise error correction for small volumes; FWE-wbc $=$ wholebrain family-wise error corrected; GMV = gray matter volume; $\mathrm{HC}=$ hippocampus; $\mathrm{MCC}=$ midcingulate cortex; $\mathrm{MNI}=$ Montreal Neurologic Institute; $\mathrm{PCC}=$ posterior cingulate cortex; ROI = region of interest; $\mathrm{VBM}=$ voxel-based morphometry

AFFILIATIONS: From the Department of Systems Neuroscience (Mr Esser and Drs Stoeckel and von Leupoldt), University Medical Center Hamburg-Eppendorf, Hamburg, Germany; Pulmonary Research Institute at LungClinic Grosshansdorf (Drs Kirsten, Watz, and Magnussen), Airway Research Center North, Member of the German Center for Lung Research, Grosshansdorf, Germany; AtemReha GmbH (Dr Taube and Ms Lehmann), Hamburg, Germany; Institute for Health and Behaviour (Dr Petersen), Research Unit
INSIDE, University of Luxembourg, Luxembourg City, Luxembourg and Research Group Health Psychology (Dr von Leupoldt), University of Leuven, Leuven, Belgium.

Some of the results have been presented as a poster at the European Respiratory Society International Congress, September 7, 2014, Munich, Germany.

FUNDING/SUPPORT: This study was supported by the German Research Foundation (Deutsche Forschungsgemeinschaft [DFG]) by a stipend [Heisenberg-Stipendium, LE 1843/9-2] and two grants [LE 1843/10-1, LE 1843/10-3] to Dr von Leupoldt.

CORRESPONDENCE TO: Andreas von Leupoldt, PhD, Research Group Health Psychology, University of Leuven, Tiensestraat 102, 3000 Leuven, Belgium; e-mail: andreas.vonleupoldt@ppw.kuleuven.be

Copyright (C) 2016 American College of Chest Physicians. Published by Elsevier Inc. All rights reserved.

DOI: http://dx.doi.org/10.1378/chest.15-0027 
COPD is a worldwide leading cause of morbidity and mortality, characterized by progressive and persistent airflow limitation caused by intrapulmonary inflammatory processes. ${ }^{1}$ The cardinal symptom is dyspnea (breathlessness), which usually presents during low-intensity activities such as climbing stairs or walking. ${ }^{1,2}$ With longer disease duration, dyspnea intensifies and can be present even at rest. Patients with COPD commonly experience dyspnea as highly aversive and threatening. ${ }^{3}$ Not surprisingly, COPD is often accompanied by high levels of comorbid anxiety and depression. ${ }^{4}$ In particular, disease-specific fears, like fear of dyspnea and fear of physical activity, contribute to disability in COPD and have been suggested to fuel a downward spiral of avoidance of physical activity, ${ }^{5}$ subsequent deconditioning, and greater dyspnea at even lower activity levels resulting in significant reductions in health-related quality of life. , $6-8^{-}$

Unfortunately, little is known about underlying brain processes in COPD. Initial studies suggested no generalized cerebral atrophy, ${ }^{9}$ but regional structural brain changes in patients with COPD, including impaired white matter microstructural integrity. ${ }^{9,10}$ However, results concerning gray matter volume (GMV) remain sparse and partly conflicting. ${ }^{11,12}$ Notably, GMV reductions previously observed in patients with COPD included limbic and paralimbic brain areas such as cingulate cortex, insula, hippocampus (HC), and amygdala (AMYG), ${ }^{10,12}$ which are not only involved in the processing of dyspnea, ${ }^{13,14}$ but also in fear/anxiety and fear-avoidance behavior, ${ }^{15-17}$ and in antinociception, that is, the downregulation of aversive nociceptive stimuli. ${ }^{18}$ Comparable GMV reductions were demonstrated in patients chronically experiencing other aversive sensations, especially pain, ${ }^{19-23}$ and these were associated with longer pain duration. ${ }^{19,21,22}$ In patients with COPD, only one previous study suggested comparable GMV changes in relation to disease duration. ${ }^{10}$ Studies examining associations between GMV, fear of dyspnea, and fear of physical activity in COPD are missing, thus limiting our understanding of the interrelationships between brain pathology and COPD-specific fears that might contribute to the course of disease by influencing patient behaviors.

By using voxel-based morphometric (VBM) analysis of MRI images, this study examined whether patients with COPD exhibit generalized cortical degeneration as well as reduced regional GMV compared with matched healthy control subjects. Moreover, we investigated the relationships between GMV and disease duration, fear of dyspnea, and fear of physical activity.

\section{Materials and Methods \\ Participants}

Thirty stable outpatients with moderate to severe COPD (GOLD [Global Initiative for Chronic Obstructive Lung Disease] stage II and III) ${ }^{1}$ were recruited at the Pulmonary Research Institute (LungClinic Grosshansdorf; $\mathrm{n}=20$ ) and at an outpatient pulmonary rehabilitation center (Atem-Reha Hamburg; $\mathrm{n}=10$ ). Patients' demographics and medical histories, including disease duration, were obtained from their medical records. Thirty control subjects without history of respiratory disease $\left(\mathrm{FEV}_{1}\right.$ in $\%$ predicted, $\left.>80 \%\right)$, and matched for age, sex, and BMI, were recruited from local databases of the Pulmonary Research Institute (LungClinic Grosshansdorf) and the Department of Systems Neuroscience (University Medical Center Hamburg-Eppendorf). Lung function was measured using standard spirometry. ${ }^{24}$ All participants were screened using a standardized diagnostic interview (Structured Clinical Interview for DSM-IV Axis I Disorders, Clinician Version [SCID-CV] $)^{25}$ and the Hospital Anxiety and Depression Scale (HADS) ${ }^{26}$ to exclude subjects with psychiatric or neurologic symptoms. The study was approved by the local medical ethics committees Hamburg (PV3007) and SchleswigHolstein (IV/EK/122/08), and conducted according to the principles expressed in the Declaration of Helsinki. All subjects gave written informed consent before participating

\section{Disease-Specific Fears}

Fear of dyspnea and fear of physical activity were assessed with the validated COPD Anxiety Questionnaire (CAF) ${ }^{27}$ The scales consist of five and six items, respectively, which are rated on a Likert scale from 0 to 4 . Higher summary scores for each scale represent higher levels of fear.

\section{VBM Image Acquisition}

High-resolution T1-weighted structural brain images were acquired with a 32-channel head coil with a three-dimensional-MPRAGE sequence (repetition time 2300 milliseconds, echo time 2.98 milliseconds, flip angle $9^{\circ}$, voxel size $1 \mathrm{~mm}$, field of view $256 \times 256,240$ slices) on a 3T Magnetom-TRIO MRI system (Siemens Medical Solutions).

\section{Procedure}

After MRI compatibility check, standardized instructions, and spirometry, participants filled in questionnaires. Thereafter, participants entered the MRI scanner and underwent the structural image acquisition.

\section{Data Analysis}

Group comparisons regarding baseline characteristics, fear ratings, and MRI-based brain volumetrics (ie, total intracranial volume, gray matter, white matter) were performed with two-sample $t$, MannWhitney $U$ (in case of nonnormal distributions) or $\chi^{2}$ (data in frequency form) tests. Respective MRI-based brain volumes of gray matter, white matter, and cerebrospinal fluid were calculated during the preprocessing for each subject in native space, meaning that tissue volumes are in spatial correspondence to the original data. Then, total intracranial volume was estimated by summing the volumes of gray matter, white matter, and cerebrospinal fluid. Partial correlation analyses and moderated mediation analyses were used to 
examine interrelationships between disease duration, fear of dyspnea/ physical activity, and GMV in brain areas where patients demonstrated reduced GMV (using the gray scale intensity value as an indicator of gray matter thickness from the local voxel showing the largest group difference). Data were analyzed using SPSS 22 software (IBM Corporation) using the Process macro for conditional process analyses for moderated mediation analysis. The threshold for statistical significance was set to $\alpha<0.05$.

Image analysis was carried out using the VBM8 toolbox r435 (Voxel Based Morphometry, http://dbm.neuro.uni-jena.de/vbm.html) implemented in SPM8 (Statistical Parametric Mapping, www.fil.ion. ucl.ac.uk/spm) running under Matlab2013a (The MathWorks, Inc). T1-weighted structural images were inspected by a neurologist regarding atrophy that could not be explained by normal aging and potentially indicates other degenerative processes like dementia. No participant had to be excluded. Preprocessing was performed using the diffeomorphic anatomical registration using exponentiated lie algebra (DARTEL) approach. Preprocessing steps included:

1. Centering of the origin of T1 images to anterior commissure, normalization into Montreal Neurologic Institute (MNI) space, additional use of the "thorough cleanup" procedure to remove remaining nonbrain tissue-which is particularly useful for older and therefore more atrophic brains-and segmentation into volumes of gray matter, white matter, and cerebrospinal fluid;

2. After initial affine registration, study-specific customized DARTEL templates were created from the entire image dataset;

3. Then, original T1 images were normalized to created DARTEL templates and modulated "nonlinearly only" to correct for nonlinear warping during spatial normalization and for differences in individual brain size to ensure that relative volumes were preserved, meaning that volumes are corrected for different brain sizes;

4. Data quality check and check for sample homogeneity using covariance was performed on gray matter images. None of the tests revealed outliers;

5. Afterward, preprocessed gray matter images were smoothed with a Gaussian kernel of full width at half maximum of $6 \mathrm{~mm}$ in all three directions.

In a first step, a whole-brain analysis with family-wise error-corrected (FWE-wbc) threshold of $P<.05$ was used to examine significant clusters of GMV differences between groups. In a second step, a small-volume analysis with a family-wise error correction (FWEsvc, $P<.05)$ was used within a priori-determined bilateral regions of interest (ROI). ROIs were chosen according to (1) previous knowledge on regional GMV reductions in patients with COPD,,$^{10,12}$ (2) structural brain changes in chronic pain syndromes, ${ }^{19-23}$ and (3) functional relevance of areas in the processing of dyspnea, ${ }^{13,14}$ fear/anxiety, and fear-avoidance behavior, ${ }^{15-17}$ and the transmission/regulation of nociceptive input. ${ }^{18,28}$ Specifically, analyzed ROIs consisted of the anterior cingulate cortex (ACC), midcingulate cortex (MCC), insula, HC, thalamus, and AMYG. ROI masks were derived from the automated anatomic labeling atlas. ${ }^{29}$ ROI analyses were performed for bilateral brain structures and were limited to a restricted
TABLE 1 ] Baseline Characteristics

\begin{tabular}{|c|c|c|}
\hline Characteristics & $\begin{array}{l}\text { Patients With } \\
\text { COPD }\end{array}$ & Control Group \\
\hline Subjects, No. & 30 & 30 \\
\hline Women (men), No. & $14(16)$ & $14(16)$ \\
\hline Age, y & $66.3(8.8)$ & $64.6(7.8)$ \\
\hline Height, cm & $170.5(9.2)$ & $172.4(9.2)$ \\
\hline Weight, kg & $76.1(12.4)$ & $76.7(15.8)$ \\
\hline BMI, $\mathrm{kg} / \mathrm{m}^{2}$ & $26.2(4.1)$ & $25.6(3.8)$ \\
\hline $\mathrm{FEV}_{1}, \%$ predicted & $49.7(12.3)$ & $121.5(13.1)^{a}$ \\
\hline FVC, \% predicted & $95.7(17.9)$ & $125.8(14.9)^{a}$ \\
\hline $\begin{array}{l}\mathrm{FEV}_{1} / \mathrm{FVC} \\
\quad \% \text { predicted }\end{array}$ & $42.0(9.0)$ & $78.4(5.0)^{a}$ \\
\hline $\begin{array}{l}\text { Medication use for } \\
\text { COPD, No. (\%) }\end{array}$ & $30(100)$ & $\ldots$ \\
\hline $\begin{array}{c}\text { Long-acting } \\
\beta \text {-agonist }\end{array}$ & $16(53)$ & $\ldots$ \\
\hline $\begin{array}{l}\text { Long-acting } \\
\text { muscarinic } \\
\text { antagonist }\end{array}$ & $28(93)$ & $\ldots$ \\
\hline \multicolumn{3}{|l|}{$\begin{array}{c}\text { Smoking status, } \\
\text { No. (\%) }\end{array}$} \\
\hline Current smokers & $15(50)$ & $2(7)^{b}$ \\
\hline Former smokers & $15(50)$ & $8(27)^{c}$ \\
\hline Never smokers & $0(0)$ & $20(66)^{a}$ \\
\hline $\begin{array}{c}\text { Disease severity, } \\
\text { moderate } \\
\text { (severe) }\end{array}$ & 11 (19) & $\ldots$ \\
\hline Disease duration, y & $9.9(5.5)$ & $\ldots$ \\
\hline $\begin{array}{l}\text { Fear of physical } \\
\text { activity, CAF }\end{array}$ & $5.8(2.1)$ & $2.2(3.0)^{a}$ \\
\hline Fear of dyspnea, CAF & $3.2(2.5)$ & $1.1(1.9)^{a}$ \\
\hline
\end{tabular}

Data are given as mean (SD) unless otherwise indicated. CAF = COPD Anxiety Questionnaire.

${ }^{a} P<.001$ for the comparison between COPD and control group.

${ }^{\mathrm{b}} P<.01$ for the comparison between COPD and control group.

${ }^{\mathrm{c}} P<.05$ for the comparison between COPD and control group.

number of predefined ROIs, while not using further correction for multiple comparisons. Differences in regional GMV between patient and control group were analyzed via two-sample $t$ tests. For the combined visualization of significant findings across different ROIs (necessitating different local thresholds based on the size of the area to determine significance), all results of GMV changes are displayed at a more liberal uncorrected $P<.001$.

\section{Results}

\section{Participants}

Means and SDs of baseline characteristics are reported in Table 1. Patients with COPD and control subjects did not differ significantly regarding age, sex, height, weight, and BMI. As expected, the COPD group showed lower lung function in $\mathrm{FEV}_{1} \%$ predicted, FVC $\%$ predicted, and $\mathrm{FEV}_{1} / \mathrm{FVC}$ (all $P<.001$ ), and contained more smokers compared with the control group $(P<.001)$. All participants were within the normal range of HADS anxiety and depression scores, that is, below the clinically relevant cutoff. Patients with COPD showed significantly higher ratings of 
TABLE 2 ] MRI-Based Brain Volumetrics

\begin{tabular}{l|c|c|c|c}
\hline Volume, $\mathrm{cm}^{3}$ & Patients With COPD & Control Group & $t$ Score & $P$ Value \\
\hline Total intracranial & $1,391.566(124.5)$ & $1,429.436(133.4)$ & -1.1 & .260 \\
Gray matter & $567.778(52.3)$ & $582.737(61.6)$ & -1.0 & .315 \\
White matter & $551.604(58.8)$ & $572.530(71.2)$ & -1.2 & .220 \\
\hline
\end{tabular}

Data are given as mean (SD).

fear of dyspnea $(P<.001)$ and fear of physical activity $(P<.001)$.

\section{MRI-Based Brain Volumetrics}

Global volumetric analysis revealed that neither total intracranial volume nor total volume of gray and white matter was significantly different between the COPD group and the control group (Table 2).

\section{VBM-Based Regional Gray Matter Changes}

For VBM, two-sample $t$ tests between groups included smoking status (current, former, and never smokers) as covariate of no interest because of potential effects of smoking on brain tissue volumes. ${ }^{30}$ Whole-brain analysis revealed that compared with control subjects, GMV in patients with COPD was reduced in posterior cingulate cortex (PCC; FWE-wbc, $P=.033$, cluster size, 639 voxels). Small-volume corrected analyses for predefined ROIs revealed decreased GMV in patients with COPD in MCC (FWE-svc, $P=.012$ ), ACC (FWE-svc, $P=.042$ ), HC (FWE-svc, $P=.021$ ), and AMYG (FWE-svc, $P=.037$ ) (Fig 1, Table 3). No differences in GMV between the patient and the control group were found within the insula and thalamus. No brain areas were found in which patients with COPD showed higher GMV compared with control subjects.

\section{Correlation and Mediation Analyses}

In patients with COPD, partial correlation (controlling for smoking status) revealed a significant negative correlation of disease duration $(r=-0.59, P=.002)$, fear
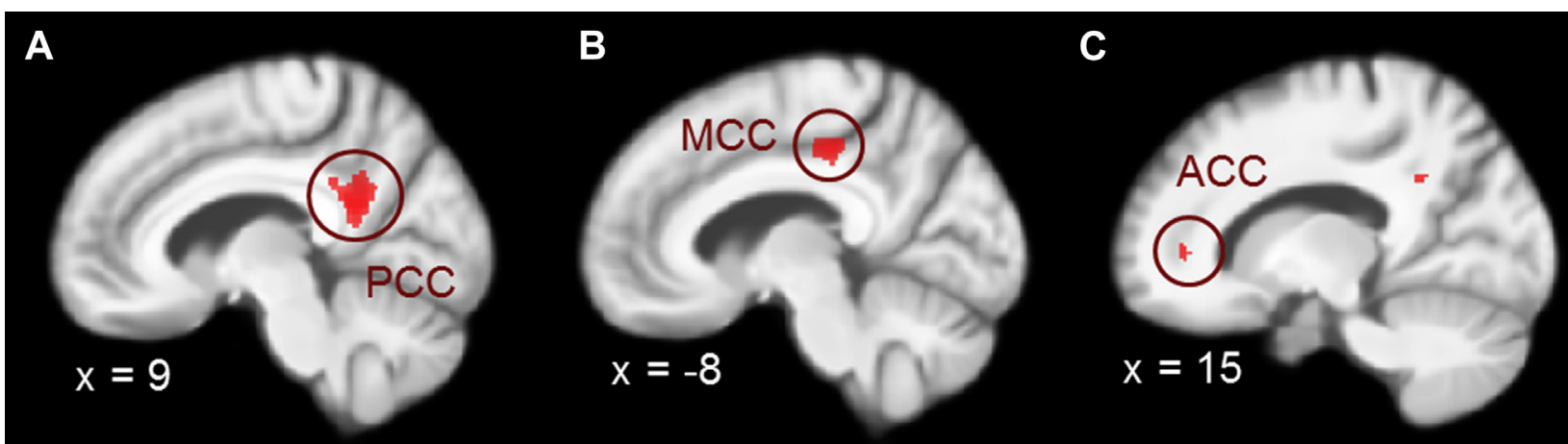

D

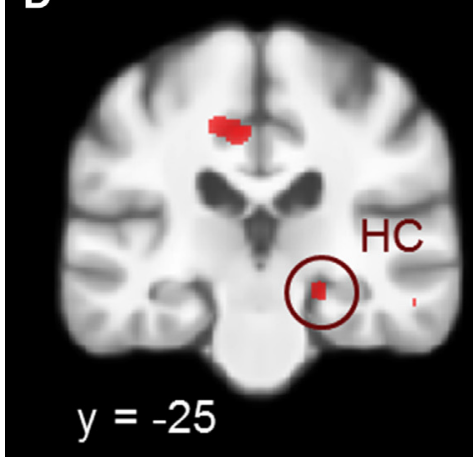

E

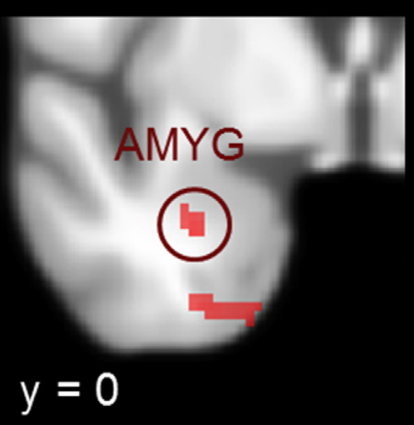

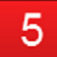

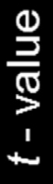

Figure $1-A-E$, Reduced gray matter volume (GMV) in patients with COPD compared with control subjects in (A) PCC (whole-brain analysis), (B) MCC (small-volume analysis), (C) ACC (small-volume analysis), (D) HC (small-volume analysis), and (E) AMYG (small-volume analysis). Results are superimposed on the mean diffeomorphic anatomical registration using exponentiated lie algebra (DARTEL)-template image and displayed at a more liberal threshold (uncorrected $\mathrm{P}<.001$ ) than statistical analyses to facilitate visualization. ACC = anterior cingulate cortex; AMYG = amygdala; $H C=$ hippocampus; $M C C=$ midcingulate cortex; $P C C=$ posterior cingulate cortex . 
TABLE 3 ] Regional Reductions in GMV in Patients With COPD Compared With Control Subjects

\begin{tabular}{|c|c|c|c|c|c|c|}
\hline \multirow[b]{2}{*}{ Anatomic Location } & \multirow[b]{2}{*}{$\mathrm{R} / \mathrm{L}$} & \multicolumn{3}{|c|}{ MNI } & \multirow[b]{2}{*}{ z Score } & \multirow[b]{2}{*}{$P$ Value } \\
\hline & & $x$ & y & z & & \\
\hline Posterior cingulate cortex & $\mathrm{R}$ & 9 & -48 & 22 & 4.81 & $.033^{a}$ \\
\hline Midcingulate cortex & $\mathrm{L}$ & -8 & -27 & 43 & 4.17 & $.012^{\mathrm{b}}$ \\
\hline Anterior cingulate cortex & $\mathrm{R}$ & 15 & 42 & 3 & 3.70 & $.042^{\mathrm{b}}$ \\
\hline Hippocampus & $\mathrm{R}$ & 21 & -25 & -12 & 3.84 & $.021^{\mathrm{b}}$ \\
\hline Amygdala & $\mathrm{L}$ & -30 & 0 & -29 & 3.28 & $.037^{\mathrm{b}}$ \\
\hline
\end{tabular}

$z$ values and $P$ values for significant gray matter volume decreases. GMV = gray matter volume; $L=$ left hemisphere; $M N I=$ Montreal Neurological Institute, peak coordinates; $\mathrm{R}=$ right hemisphere.

ahole-brain family-wise error corrected.

bSmall-volume family-wise error corrected for bilateral region of interest.

of dyspnea $(r=-0.53, P=.005)$, and fear of physical activity $(r=-0.46, P=.019)$ with GMV in the ACC (ACC-GMV), but not with GMV in MCC, PCC, HC, and AMYG (Fig 2). Disease duration was further significantly correlated with fear of physical activity $(r=0.49$,

$P=.011)$, but not with fear of dyspnea $(r=0.26, P=.20)$. The correlation between fear of physical activity and fear of dyspnea was not significant $(r=0.21, P=.31)$.

Subsequently, a moderated mediation analysis tested whether the relationship between disease duration and ACC-GMV was mediated by disease-specific fears. Again, smoking status was controlled for by including it as moderator in the model (Fig 3). The moderated mediation model (excluding the association of ACC-GMV and fear of dyspnea) reached statistical significance with an explained variance of $R^{2}=0.46$ $(P=.02)$. Specifically, fear of physical activity determined a significant proportion of the relationship between disease duration and ACC-GMV $\left(\Delta R^{2}=0.17\right)$.

Explorative post hoc analyses tested for potential additional interactions with patient age. Partial correlations (controlling for smoking status) between patient age and disease duration $(r=0.20, P=.33)$ and disease-specific fears (fear of physical activity: $r=0.34$, $P=.09$; fear of dyspnea: $r=0.10, P=.62$ ) remained nonsignificant. When calculating the moderated mediation model by controlling for age in addition to smoking status, the model again reached statistical significance with an explained variance of $R^{2}=0.62$ $(P=.011)$. This time, fear of physical activity determined a significant proportion of the relationship between disease duration and ACC-GMV of $\Delta R^{2}=0.26$.

\section{Discussion}

This study is one of the first to investigate structural brain changes in patients with COPD using VBM and several important findings were obtained. Patients with COPD showed no general reductions in MRI brain volume of gray matter, white matter, or total intracranial volume compared with matched healthy control subjects. In contrast, patients with COPD showed regionally decreased GMV within the PCC (whole-brain analysis) as well as in ACC, MCC, HC, and AMYG (small-volume analysis) when compared with control subjects. Most importantly, we observed that reductions in GMV in the ACC were not only correlated with longer disease duration, but also with higher levels of fear of dyspnea and fear of physical activity. No such relationships were observed in the healthy control group. Together, the results firstly link structural brain changes in COPD with increased disease-specific fears, which might negatively impact the course of the disease via behavioral mechanisms as will be discussed later.

The present findings contribute to the limited literature on COPD brain pathology in several ways. First, comparable overall MRI brain volumes of gray matter, white matter, and total intracranial volume in patients with COPD and healthy control subjects suggest an absence of generalized cerebral degeneration in patients with COPD. This supports findings by Dodd et $\mathrm{al}^{9}$ who reported comparable volumes of gray and white matter in patients with COPD and a healthy control group. Second, our observation of regionally decreased GMV in patients with COPD within the ACC, MCC, PCC, HC, and AMYG substantiates the preliminary observations of Zhang et al. ${ }^{10}$ Using VBM, these authors found regionally decreased gray matter in comparable brain regions which further included insula and thalamus. The findings in the latter two areas could not be replicated in the present study. Importantly, like Zhang et al, ${ }^{10}$ we found a negative correlation between GMV reductions in the ACC and disease duration, suggesting stronger ACC degeneration with increasing chronicity of COPD. 
Hippocampal volume reductions in patients with COPD were also reported by $\mathrm{Li}$ and $\mathrm{Fei}^{12}$ using different methodology, but not by Borson et al, ${ }^{11}$ based, however, on a limited number of participants. Notably, ACC, MCC, PCC, HC, and AMYG are not only involved in the neural processing of dyspnea, ${ }^{13,14}$ but also highly relevant for the processing of fear/anxiety and fearavoidance behavior. ${ }^{15-17,31}$ This already suggests a potential link between GMV changes in COPD and behavioral aspects such as fear.

Most importantly, we firstly demonstrated that structural brain changes in patients with COPD were related to disease-specific fears. Specifically, reduced

A

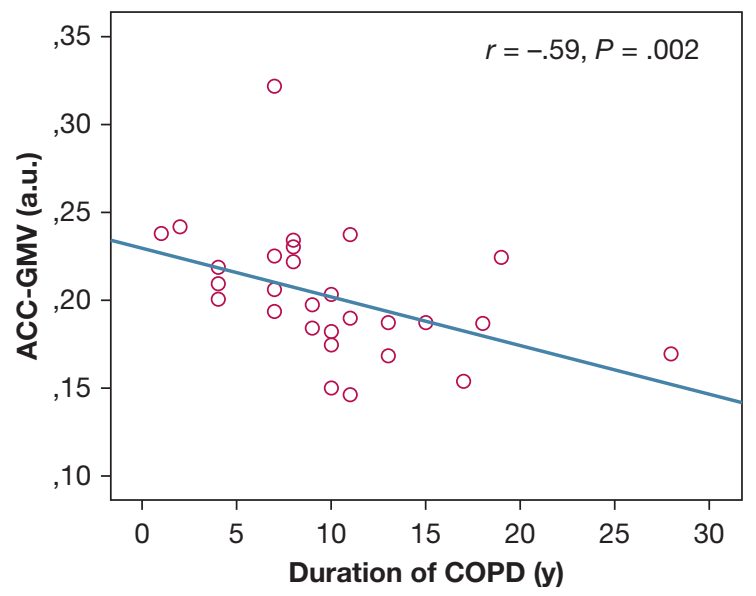

C

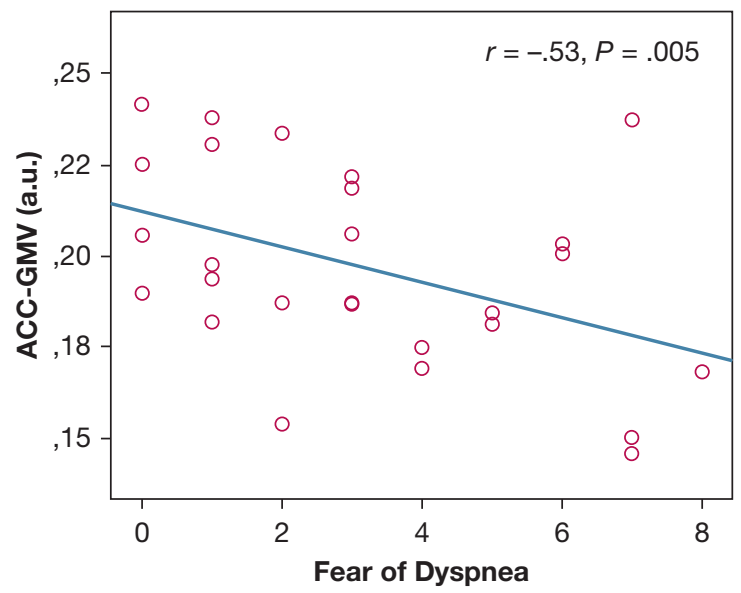

GMV in the ACC in patients with COPD was correlated with greater fear of dyspnea and fear of physical activity. This converges with the well-known role of the ACC for the processing of aversive stimuli including dyspnea, ${ }^{13,14}$ pain, ${ }^{19,31,32}$ and fear. ${ }^{16}$ Moreover, the ACC is a central component in emotion regulation such as fearavoidance behavior ${ }^{17,33}$ and appraisal of threat stimuli including respiratory threats. ${ }^{13,31}$ Notably, the ACC also plays an important role in the descending antinociceptive network mediating the analgetic effects of opiates and placebo interventions on perceived pain. ${ }^{34,35}$ Gray matter decreases within the ACC were reported for diverse chronic pain syndromes, ${ }^{23}$ and shown to be related to the chronicity of disease and the

B
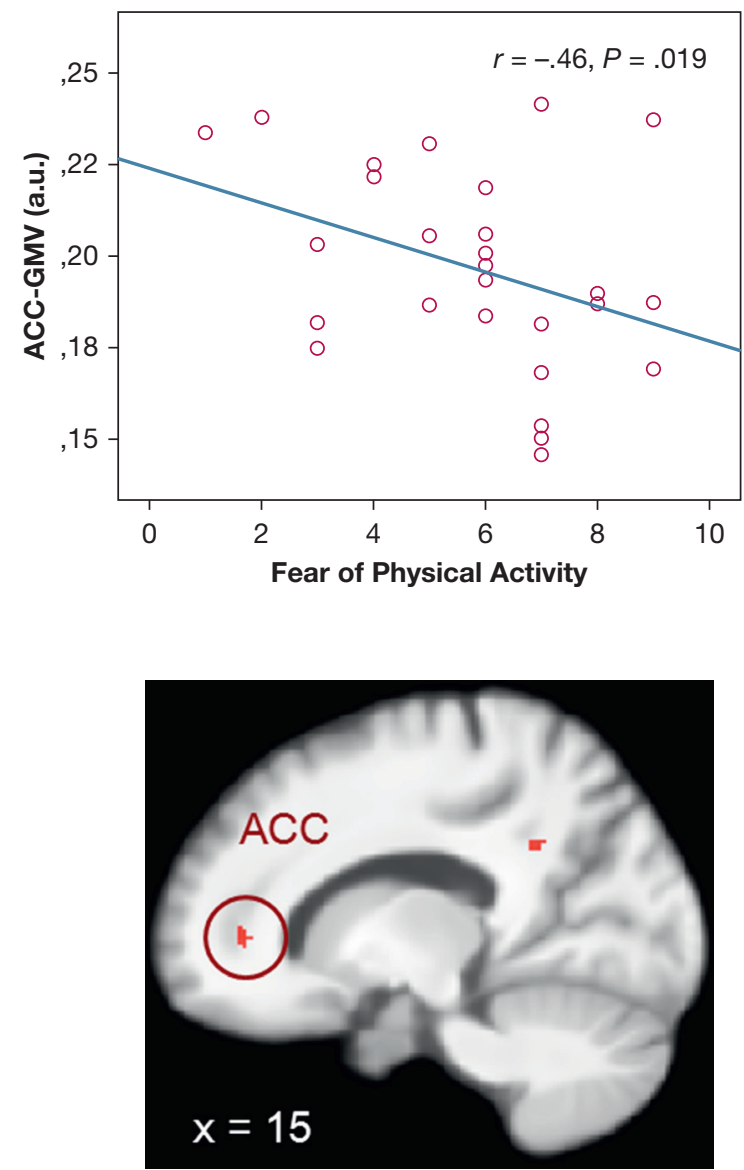

Figure $2-A-C$, Negative correlations of ACC-GMV in patients with COPD with (A) disease duration of COPD, (B) fear of physical activity, and (C) fear of dyspnea. Specifically, correlations are depicted for the GMV from the local voxel within the circled ACC, in which patients showed the largest difference compared with control subjects (voxel Montreal Neurological Institute [MNI] coordinates: $x=15, y=42, z=3$ ). Results are superimposed on the mean DARTEL-template image and displayed at a more liberal threshold (uncorrected $\mathrm{P}<.001$ ) than statistical analyses to facilitate visualization. ACC-GMV = gray matter volume in the anterior cingulate cortex; a.u. = arbitrary unit. See Figure 1 legend for expansion of other abbreviations. 
Figure 3 - Moderated mediation model with partial correlation coefficients (controlled for smoking status) in patients with COPD. See Figure 2 legend for expansion of abbreviations.

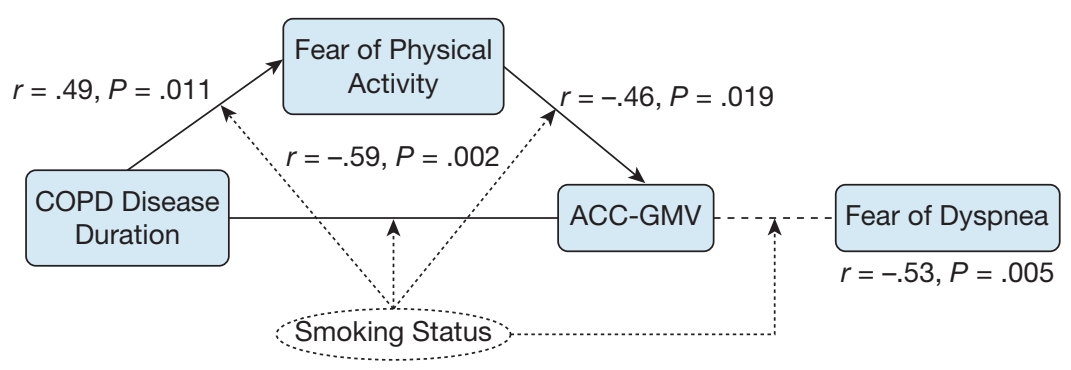

intensity of noxious stimulation. ${ }^{36-38}$ This converges with the negative correlation of ACC-GMV and disease duration observed in this study. Degeneration of the ACC due to the chronic experience of dyspnea might, in turn, result in reduced antinociceptive impact of the ACC, thereby increasing the fear of dyspnea and fear of physical activity.

Finally, mediation analyses demonstrated that the relation of ACC-GMV decreases and disease duration was mediated by higher fear of physical activity.

This suggests that longer disease duration, even after controlling for the potentially confounding effects of smoking and age, results in increased fear of physical activity, which may subsequently lead to avoidance of physical activity and contribute to subsequent GMV loss in the ACC. Previous studies have convincingly demonstrated that reduced physical activity is related to GMV loss. ${ }^{39,40}$ Increased fear of dyspnea due to lower ACC functionality might further fuel this vicious circle and lead to even more fear and avoidance of physical activity, subsequent deconditioning, increased dyspnea, and greater reductions in health-related quality of life. ${ }^{5,6}$ Addressing these disease-specific fears seems, therefore, highly important in the treatment of COPD. ${ }^{5,6}$ In this regard, it would be interesting to examine whether the treatment of dyspnea and disease-specific fears can stop or even reverse GMV reductions in patients with COPD. Previous findings in chronic pain patients demonstrated that pain-related gray matter changes are not static and at least partially reversible after successful treatment of pain. ${ }^{41-43}$

Potential limitations of the present study include the cross-sectional character and the use of questionnaire data to assess fear and potential avoidance of physical activity. Therefore, future studies including longitudinal designs and objective measures of physical activity such as activity monitors are warranted to gain further insights into the dynamics of GMV changes in relation to activity avoidance in COPD. Moreover, the specific physiologic mechanisms underlying the observed GMV changes in patients with COPD remain unclear. Various mechanisms including axonal remodeling, changes in dendritic spines, dendritic atrophy, synaptic plasticity (eg, synaptogenesis, synapse elimination, long-term potentiation/depression, or synapse turnover), changes in cell size, changes in oxygenation, and neural or glial cell genesis have been suggested to contribute to experience-dependent cortical plasticity. ${ }^{44-50}$ Therefore, future studies with different methodology will be necessary to examine which of these potential mechanisms contribute to structural brain changes in COPD.

\section{Conclusions}

Compared with healthy control subjects, patients with COPD showed decreased GMV in ACC, MCC, and PCC, HC, and AMYG in the absence of generalized cortical degeneration. The affected brain areas are involved in the processing of dyspnea, fear, and antinociception. Moreover, decreased gray matter in the ACC in patients with COPD was related to longer disease duration, and greater fear of dyspnea and fear of physical activity, which-via behavioral mechanismsmight negatively influence the course of disease. Targeting these disease-specific fears in patients with COPD might not only improve outcomes of clinical interventions such as pulmonary rehabilitation, but also reverse structural brain changes in these patients. 


\section{Acknowledgments}

Author contributions: R. W. E. and A. v. L. had full access to all of the data in the study and take full responsibility for the integrity of the data and accuracy of the data analysis, including and especially any adverse effects. R. W. E., M. C. S., H. M., and A. v. L. contributed to study conception and design; R. W. E., M. C. S., A. K., H. W., K. T., and K. L. contributed to data acquisition; R. W. E., M. C. S., S. P., and A. v. L. contributed to data analysis and interpretation of data; R. W. E., M. C. S., and A. v. L. contributed to the drafting of the manuscript; and R. W. E., M. C. S., A. K., H. W., K. T., K. L., S. P., H. M., and A. v. L. contributed to manuscript revision.

\section{Conflict of interest: None declared.}

Role of sponsors: The funder had no role in the design of the study, the collection and analysis of the data, or the preparation of the manuscript.

Other contributions: We thank Timo Kraemer, MTRA, Katrin Bergholz, MTRA, Kathrin Wendt, MTRA, Friederike Behmer, MTRA, and Nergiz Turgut, MTRA, for technical assistance during data acquisition. We also thank the members of the Pulmonary Research Institute at LungClinic Grosshansdorf and Atem-Reha $\mathrm{GmbH}$ for their support in identifying patients for this study and preexamination, and especially all volunteers for participation.

\section{References}

1. Global Initiative for Chronic Obstructive Lung Disease (GOLD). Global strategy for the diagnosis, management and prevention of COPD. 2014. GOLD website. http://www.goldcopd.org/. Accessed October 10, 2014.

2. O'Donnell DE, Banzett RB, CarrieriKohlman V, et al. Pathophysiology of dyspnea in chronic obstructive pulmonary disease: a roundtable. Proc Am Thorac Soc. 2007;4(2):145-168.

3. Parshall MB, Schwartzstein RM, Adams L, et al; American Thoracic Society Committee on Dyspnea. An official American Thoracic Society statement: update on the mechanisms, assessment, and management of dyspnea. Am J Respir Crit Care Med. 2012;185(4):435-452.

4. Maurer J, Rebbapragada V, Borson S, et al; ACCP Workshop Panel on Anxiety and Depression in COPD. Anxiety and depression in COPD: current understanding, unanswered questions, and research needs. Chest. 2008;134(suppl 4):43S-56S

5. Keil DC, Stenzel NM, Kühl K, et al. The impact of chronic obstructive pulmonary disease-related fears on disease-specific disability. Chron Respir Dis. 2014;11(1): 31-40.

6. Hayen A, Herigstad M, Pattinson KTS. Understanding dyspnea as a complex individual experience. Maturitas. 2013;76(1):45-50.
7. Janssens T, De Peuter S, Stans L, et al Dyspnea perception in COPD: association between anxiety, dyspnea-related fear, and dyspnea in a pulmonary rehabilitation program. Chest. 2011;140(3):618-625.

8. von Leupoldt A, Kenn K. The psychology of chronic obstructive pulmonary disease. Curr Opin Psychiatry. 2013;26(5):458-463.

9. Dodd JW, Chung AW, van den Broek MD, Barrick TR, Charlton RA, Jones PW. Brain structure and function in chronic obstructive pulmonary disease: a multimodal cranial magnetic resonance imaging study. $A m$ Respir Crit Care Med. 2012;186(3):240-245.

10. Zhang H, Wang X, Lin J, et al. Grey and white matter abnormalities in chronic obstructive pulmonary disease: a casecontrol study. BMJ Open. 2012;2(2): e000844.

11. Borson S, Scanlan J, Friedman S, et al. Modeling the impact of COPD on the brain. Int J Chron Obstruct Pulmon Dis. 2008;3(3):429-434.

12. Li J, Fei G-H. The unique alterations of hippocampus and cognitive impairment in chronic obstructive pulmonary disease. Respir Res. 2013;14:140.

13. Davenport PW, Vovk A. Cortical and subcortical central neural pathways in respiratory sensations. Respir Physiol Neurobiol. 2009;167(1):72-86.

14. Herigstad M, Hayen A, Wiech K, Pattinson KTS. Dyspnoea and the brain. Respir Med. 2011;105(6):809-817.

15. Mechias M-L, Etkin A, Kalisch R. A meta-analysis of instructed fear studies: implications for conscious appraisal of threat. Neuroimage. 2010;49(2):1760-1768.

16. Sehlmeyer C, Schöning S, Zwitserlood P et al. Human fear conditioning and extinction in neuroimaging: a systematic review. PLoS One. 2009;4(6):e5865.

17. Wiech K, Tracey I. Pain, decisions, and actions: a motivational perspective. Front Neurosci. 2013;7:46

18. Tracey I, Mantyh PW. The cerebral signature for pain perception and its modulation. Neuron. 2007;55(3):377-391.

19. Apkarian AV, Hashmi JA, Baliki MN Pain and the brain: specificity and plasticity of the brain in clinical chronic pain. Pain. 2011;152(suppl 3):S49-S64.

20. Baliki MN, Schnitzer TJ, Bauer WR, Apkarian AV. Brain morphological signatures for chronic pain. PLoS One. 2011;6(10):e26010.

21. May A. Chronic pain may change the structure of the brain. Pain. 2008;137(1): 7-15.

22. May A. Structural brain imaging: a window into chronic pain. Neuroscientist. 2011;17(2):209-220.

23. Smallwood RF, Laird AR, Ramage AE, et al. Structural brain anomalies and chronic pain: a quantitative meta-analysis of gray matter volume. J Pain. 2013;14(7):663-675.

24. Miller MR, Hankinson J, Brusasco V, et al; ATS/ERS Task Force. Standardisation of spirometry. Eur Respir J. 2005;26(2): 319-338.
25. First MB, Spitzer RL, Gibbon M, Williams JBW. Structured Clinical Interview for DSM-IV Axis I Disorders, Clinician Version (SCID-CV). Washington, DC: American Psychiatric Press, Inc; 1996.

26. Herrmann C, Buss U, Snaith RP. Hospital Anxiety and Depression Scale - Deutsche Version (HADS - D) Manual. Bern, Switzerland: Hans Huber; 1995.

27. Kühl K, Kuhn C, Kenn K, Rief W. The COPD-Anxiety-Questionnaire (CAF): a new instrument to assess illness specific anxiety in COPD patients [in German]. Psychother Psychosom Med Psychol. 2011;61(1):e1-e9.

28. Mansour AR, Farmer MA, Baliki MN, Apkarian AV. Chronic pain: the role of learning and brain plasticity. Restor Neurol Neurosci. 2014;32(1):129-139.

29. Tzourio-Mazoyer N, Landeau B, Papathanassiou D, et al. Automated anatomical labeling of activations in SPM using a macroscopic anatomical parcellation of the MNI MRI single-subject brain. Neuroimage. 2002;15(1):273-289.

30. Liao Y, Tang J, Liu T, Chen X, Hao W. Differences between smokers and nonsmokers in regional gray matter volumes: a voxel-based morphometry study. Addict Biol. 2012;17(6):977-980.

31. Vogt BA. Pain and emotion interactions in subregions of the cingulate gyrus. Nat Rev Neurosci. 2005;6(7):533-544.

32. Apkarian AV, Bushnell MC, Treede R-D, Zubieta J-K. Human brain mechanisms of pain perception and regulation in health and disease. Eur J Pain. 2005;9(4):463-484.

33. Etkin A, Egner T, Kalisch R. Emotional processing in anterior cingulate and medial prefrontal cortex. Trends Cogn Sci. 2011;15(2):85-93.

34. Bingel U, Lorenz J, Schoell E, Weiller C, Büchel C. Mechanisms of placebo analgesia: rACC recruitment of a subcortical antinociceptive network. Pain. 2006;120(1-2):8-15.

35. Tracey I. Imaging pain. Br J Anaesth. 2008;101(1):32-39.

36. Kim JH, Suh S-I, Seol HY, et al. Regional grey matter changes in patients with migraine: a voxel-based morphometry study. Cephalalgia. 2008;28(6):598-604.

37. Jensen KB, Srinivasan P, Spaeth R, et al. Overlapping structural and functional brain changes in patients with long-term exposure to fibromyalgia pain. Arthritis Rheum. 2013;65(12):3293-3303.

38. Schmidt-Wilcke T, Leinisch E, Straube A, et al. Gray matter decrease in patients with chronic tension type headache. Neurology. 2005;65(9):1483-1486.

39. Boyle CP, Raji CA, Erickson KI, et al. Physical activity, body mass index, and brain atrophy in Alzheimer's disease. Neurobiol Aging. 2015;36(suppl 1):S194-S202.

40. Prakash RS, Snook EM, Motl RW, Kramer AF. Aerobic fitness is associated with gray matter volume and white matter integrity in multiple sclerosis. Brain Res. 2010;1341:41-51. 
41. Obermann M, Nebel K, Schumann C, et al. Gray matter changes related to chronic posttraumatic headache. Neurology. 2009;73(12):978-983.

42. Ruscheweyh R, Deppe M, Lohmann H, et al. Pain is associated with regional grey matter reduction in the general population. Pain. 2011;152(4):904-911.

43. Rodriguez-Raecke R, Niemeier A, Ihle K Ruether W, May A. Structural brain changes in chronic pain reflect probably neither damage nor atrophy. PLoS One. 2013;8(2):e54475.
44. Barnes SJ, Finnerty GT. Sensory experience and cortical rewiring. Neuroscientist. 2010;16(2):186-198.

45. Gage FH. Neurogenesis in the adult brain. J Neurosci. 2002;22(3):612-613.

46. May A, Hajak G, Gänssbauer S, et al. Structural brain alterations following 5 days of intervention: dynamic aspects of neuroplasticity. Cereb Cortex. 2007;17(1): 205-210.

47. Muotri AR, Gage FH. Generation of neuronal variability and complexity. Nature. 2006;441(7097):1087-1093.
48. Ortapamuk H, Naldoken S. Brain perfusion abnormalities in chronic obstructive pulmonary disease: comparison with cognitive impairment. Ann Nucl Med. 2006;20(2):99-106.

49. Trachtenberg JT, Chen BE, Knott GW, et al. Long-term in vivo imaging of experiencedependent synaptic plasticity in adult cortex. Nature. 2002;420(6917):788-794.

50. Yalcin I, Barthas F, Barrot M. Emotional consequences of neuropathic pain: insight from preclinical studies. Neurosci Biobehav Rev. 2014;47:154-164. 